\title{
MODULI OF ISOLATED HYPERSURFACE SINGULARITIES *
}

\author{
MICHAEL G. EASTWOOD ${ }^{\dagger}$
}

\begin{abstract}
It was shown by J.N. Mather and S.S.-T. Yau that an isolated complex hypersurface singularity is completely determined by its moduli algebra. In this article it is shown, for the simple elliptic singularities, how to construct continuous invariants from the moduli algebras and, hence, associate invariants to the singularities themselves.
\end{abstract}

1. Introduction. Suppose $V$ is a complex hypersurface in $\mathbb{C}^{n}$ with an isolated singularity at the origin. Writing $V$ as the zero locus of a holomorphic function $f\left(z_{1}, \ldots, z_{n}\right)$, it is well-known that the 'moduli algebra'

$$
A=\mathbb{C}\left\{z_{1}, \ldots, z_{n}\right\} /\left(f, \partial f / \partial z_{1}, \ldots, \partial f / \partial z_{n}\right)
$$

is a finite-dimensional algebra depending only on the germ of $V$ at the origin and is invariant under holomorphic change of coördinates. Remarkably, it was shown by Mather and Yau [4] that $A$ completely determines the germ of $V$. Thus, one should be able to distinguish between biholomorphically inequivalent singularities on the basis of their corresponding moduli algebras. This was accomplished for the simple elliptic singularities $\tilde{E}_{7}$ and $\tilde{E}_{8}$ by Seeley and Yau [6] and for $\tilde{E}_{6}$ by Chen, Seeley, and Yau [1]. In particular, they recovered Saito's $j$-invariant [5] for $\tilde{E}_{7}$ and $\tilde{E}_{8}$ and detected an error in his calculation for $\tilde{E}_{6}$. In [5], the $j$-invariant was defined as that associated to the elliptic curve arising as the exceptional divisor of the minimal resolution of $V$.

The analysis in $[1,6]$ is fairly involved and specific to $\tilde{E}_{6}, \tilde{E}_{7}$, and $\tilde{E}_{8}$. In this article we show how to employ classical invariant theory to obtain $j$ directly and rather quickly from $A$. We explain $\tilde{E}_{6}, \tilde{E}_{7}$, and $\tilde{E}_{8}$ in detail but the approach is quite general. To illustrate this we present another example.

I would like to thank Stephen Yau for drawing my attention to this problem and for several interesting conversations. Thanks are also due to Adam Harris for further useful conversations.

I would like to dedicate this article to Yum-Tong Siu on the occasion of his $60^{\text {th }}$ birthday.

2. Constructing Invariants. The moduli algebra $A$ evidently has a unique maximal ideal $\mathfrak{m}$ generated by the coördinate functions $z_{1}, \ldots, z_{n}$. Suppose that $\mathfrak{m}^{N}$ is one-dimensional and $\mathfrak{m}^{N+1}=0$. Then the algebra multiplication defines a linear transformation $\bigodot^{N} \mathfrak{m} / \mathfrak{m}^{2} \rightarrow \mathfrak{m}^{N}$ or, in other words, a tensor $a \in \bigodot^{N}\left(\mathfrak{m} / \mathfrak{m}^{2}\right)^{*}$, canonically defined up to scale. The special linear group $\operatorname{SL}\left(\mathfrak{m} / \mathfrak{m}^{2}\right)$ acts on $a$ and we may consider its classical invariants. By definition these are the polynomials in the coefficients of $a$ that are invariant under the action of $\mathrm{SL}\left(\mathfrak{m} / \mathfrak{m}^{2}\right)$. Weyl's classical invariant theory [7] dictates the general form of such invariants. Some discussion and examples are provided in an appendix.

THEOREM 2.1. Suppose $P$ and $Q$ are classical invariants of a and homogeneous of the same degree. Suppose $Q$ is non-zero. Then $P / Q$ is an absolute invariant of the algebra $A$. More precisely, if $B$ is a complex algebra isomorphic to $A$ then it must also be local, say with maximal ideal $\mathfrak{n}$ of the same dimension as $\mathfrak{m}$, also with

\footnotetext{
*Received July 31, 2003; accepted for publication August 19, 2003.

${ }^{\dagger}$ Pure Mathematics Department, Adelaide University, South Australia 5005, Australia (meastwoo@maths.adelaide.edu.au). This research was supported by the Australian Research Council.
} 
$\mathfrak{n}^{N}$ one-dimensional and $\mathfrak{n}^{N+1}=0$ and so that the value of $P / Q$ computed for the multiplication tensor $\bigodot^{N} \mathfrak{n} / \mathfrak{n}^{2} \rightarrow \mathfrak{n}^{N}$ is the same as that computed for $A$.

Proof. Let us write $\rho$ for the representation of $\operatorname{GL}\left(\mathfrak{m} / \mathfrak{m}^{2}\right)$ on $\bigodot^{N}\left(\mathfrak{m} / \mathfrak{m}^{2}\right)^{*}$. To say that $P(a)$ is a classical invariant of a tensor $a \in \bigodot^{N}\left(\mathfrak{m} / \mathfrak{m}^{2}\right)^{*}$ is to say that $P(a)=$ $P(\rho(M) a)$ for any $M \in \operatorname{SL}\left(\mathfrak{m} / \mathfrak{m}^{2}\right)$. Let $n=\operatorname{dim}_{\mathbb{C}}\left(\mathfrak{m} / \mathfrak{m}^{2}\right)$. If $P$ is homogeneous of degree $d$, then according to the classical theory (explained in the appendix), $n$ must divide $d N$ and $P(a)=\operatorname{det}(M)^{d N / n} P(\rho(M) a)$ for $M \in \operatorname{GL}\left(\mathfrak{m} / \mathfrak{m}^{2}\right)$. Therefore, the ratio $P(a) / Q(a)$ does not see any choice of basis. Neither does it see the scale ambiguity in $a$ : replacing $a$ by $\lambda a$ scales both $P$ and $Q$ by $\lambda^{d}$. These were the only arbitrary ingredients in the computation of $P / Q$.

This theorem is brought to life by the examples in the following section, which in turn depend on the particular classical invariants given in the appendix. There are variations on this construction in the presence of other canonically defined ideals in $A$. The singularities $\tilde{E}_{8}$ yield to such a variation.

3. Examples. The following examples are typical. The first three were studied by Saito [5].

3.1. The simple elliptic singularities $\tilde{E}_{6}$. Following [1], we write these singularities in $\mathbb{C}^{3}$ in the form

$$
V_{t}=\left\{x^{3}+y^{3}+z^{3}+t x y z=0\right\} \quad \text { for a parameter } t \text { with } t^{3}+27 \neq 0
$$

and present the moduli algebra as

$$
A_{t}=\mathbb{C}\langle 1, x, y, z, y z, z x, x y, x y z\rangle \text { where } x^{2}=-\frac{t}{3} y z, y^{2}=-\frac{t}{3} z x, z^{2}=-\frac{t}{3} x y .
$$

Then, the maximal ideal is $\mathfrak{m}=(x, y, z)$ and we may write

$$
\mathfrak{m} / \mathfrak{m}^{2}=\mathbb{C}\langle x, y, z\rangle \text { and } \quad \mathfrak{m}^{3}=\mathbb{C}\langle x y z\rangle .
$$

Multiplication $\bigodot^{3} \mathfrak{m} / \mathfrak{m}^{2} \rightarrow \mathfrak{m}^{3}$ has the following effect:-

$$
x^{3} \mapsto-\frac{t}{3} x y z \quad y^{3} \mapsto-\frac{t}{3} x y z \quad z^{3} \mapsto-\frac{t}{3} x y z \quad x y z \mapsto x y z
$$

and all other monomials are sent to zero. As a polynomial with respect to the dual basis $X, Y, Z$ of $\left(\mathfrak{m} / \mathfrak{m}^{2}\right)^{*}$, this is

$$
F_{t}(X, Y, Z)=t X^{3}+t Y^{3}+t Z^{3}-18 X Y Z
$$

up to scale. Notice that this polynomial does not define the original variety $V_{t}$. For example, it is singular if and only if $t=0$ or $t^{3}=216$. This already distinguishes these values of $t$ as special, in agreement with Chen, Seeley, and Yau [1] who observe that the Lie algebra of derivations of $A_{t}$ jumps dimension for these values of $t$. The invariant theory of homogeneous cubic polynomials in three variables in presented in the appendix. In particular, there is an invariant $J$ of degree 4 and an invariant $K$ of degree 6. A computation (easily carried out with computer algebra, as explained in the appendix) gives

$$
\frac{J^{3}-6 K^{2}}{J^{3}}=-\frac{t^{3}\left(t^{3}-216\right)^{3}}{1728\left(t^{3}+27\right)^{3}} .
$$


The expression on the right is the $j$-invariant (with Saito's normalisation [5]) associated to the elliptic curve defined by the original equation

$$
x^{3}+y^{3}+z^{3}+t x y z=0
$$

in $\mathbb{C P}_{2}$. (In the appendix, we shall explain how to compute the $j$-invariant for any non-singular cubic without prior normalisation. It is interesting to note that the elliptic curves defined in $\mathbb{C P}_{2}$ by (3.1) and (3.3) have reciprocal $j$-invariants.) This corrects Saito's computation in [5] and, multiplying by -1728 , gives the invariant found by Chen, Seeley, and Yau [1]. Once this invariant is known it is easy to see that it is complete: the coördinate changes

$$
x \mapsto \omega x \quad y \mapsto y \quad z \mapsto z
$$

and

$$
x \mapsto x+y+z \quad y \mapsto \omega x+\omega^{2} y+z \quad y \mapsto \omega^{2} x+\omega y+z,
$$

where $\omega^{3}=1$, preserve the form of $V_{t}$ but induce

$$
t \mapsto \omega t \quad \text { and } \quad t \mapsto \frac{3(6-t)}{3+t}
$$

as regards the parameter $t$. These two substitutions (3.6) generate the changes in $t$ that preserve (3.2). In fact, Chen, Seeley, and Yau [1] show that, along with simply permuting and scaling $(x, y, z)$, the substitutions (3.4) and (3.5) generate the only coördinate changes preserving the form of $V_{t}$.

The relationship between (3.3) and (3.1) is deceptively simple. If, instead, we had started with a cubic polynomial in Weierstraß normal form

$$
y^{2} z-4 x^{3}+g_{2} x z^{2}+g_{3} z^{3}
$$

then the corresponding cubic polynomial $\bigodot^{3} \mathfrak{m} / \mathfrak{m}^{2} \rightarrow \mathfrak{m}^{3}$ would be

$$
Y^{2} Z-4\left(\frac{g_{2}{ }^{3}}{27 g_{3}{ }^{2}-g_{2}{ }^{3}}\right) X^{3}+g_{2} X Z^{2}+g_{3} Z^{3}
$$

for a suitable choice of basis.

3.2. The simple elliptic singularities $\tilde{E}_{7}$. Following $[6]$, we write these singularities in $\mathbb{C}^{3}$ in the form

$$
V_{t}=\left\{x^{4}+t x^{2} y^{2}+y^{4}+z^{2}=0\right\} \quad \text { for a parameter } t \text { with } t^{2} \neq 4
$$

and present the moduli algebra as

$$
A_{t}=\mathbb{C}\left\langle 1, x, y, x^{2}, x y, y^{2}, x^{2} y, x y^{2}, x^{2} y^{2}\right\rangle \text { where } x^{3}=-\frac{t}{2} x y^{2}, y^{3}=-\frac{t}{2} x^{2} y .
$$

Then, the maximal ideal is $\mathfrak{m}=(x, y)$ and we may write

$$
\mathfrak{m} / \mathfrak{m}^{2}=\mathbb{C}\langle x, y\rangle \quad \text { and } \quad \mathfrak{m}^{4}=\mathbb{C}\left\langle x^{2} y^{2}\right\rangle .
$$

Multiplication $\bigodot^{3} \mathfrak{m} / \mathfrak{m}^{2} \rightarrow \mathfrak{m}^{4}$ has the following effect:-

$$
x^{4} \mapsto-\frac{t}{2} x^{2} y^{2} \quad x^{3} y \mapsto 0 \quad x^{2} y^{2} \mapsto x^{2} y^{2} \quad x y^{2} \mapsto 0 \quad y^{4} \mapsto-\frac{t}{2} x^{2} y^{2} .
$$


As a polynomial with respect to the dual basis $X, Y$ of $\left(\mathfrak{m} / \mathfrak{m}^{2}\right)^{*}$, this is

$$
t X^{4}-12 X^{2} Y^{2}+t Y^{4}
$$

up to scale. The invariant theory of homogeneous quartic polynomials in two variables in presented in the appendix. In particular, there is an invariant $J$ of degree 2 and an invariant $K$ of degree 3. A computation (easily carried out with computer algebra, as explained in the appendix) gives

$$
\frac{J^{3}}{6 K^{2}}=\frac{\left(12+t^{2}\right)^{3}}{108\left(t^{2}-4\right)^{2}} .
$$

The expression on the right is the $j$-invariant computed by Saito [5]. The substitutions

$$
t \mapsto-t \quad \text { and } \quad t \mapsto \frac{2(6-t)}{2+t}
$$

leave (3.8) unchanged and generate the group of such substitutions. These arise from the coördinate changes

$$
\left.\left.\begin{array}{l}
x \mapsto i x \\
y \mapsto y
\end{array}\right\} \quad \text { and } \quad \begin{array}{l}
x \mapsto x+y \\
y \mapsto x-y
\end{array}\right\},
$$

respectively. It follows that the $j$-invariant is complete. In fact, it is not hard to show that, along with simply swopping and scaling $(x, y)$, the substitutions (3.9) generate the only coördinate changes preserving the form of $V_{t}$.

3.3. The simple elliptic singularities $\tilde{E}_{8}$. Following [6], we write these singularities in $\mathbb{C}^{3}$ in the form

$$
V_{t}=\left\{x^{6}+t x^{4} y+y^{3}+z^{2}=0\right\} \quad \text { for a parameter } t \text { with } 4 t^{3}+27 \neq 0
$$

and present the moduli algebra as

$$
A_{t}=\mathbb{C}\left\langle 1, x, y, x^{2}, x y, x^{3}, x^{2} y, x^{4}, x^{3} y, x^{4} y\right\rangle \text { where } y^{2}=-\frac{t}{3} x^{4}, x^{5}=-\frac{2 t}{3} x^{3} y .
$$

There is the maximal ideal $\mathfrak{m}=(x, y)$ but also another canonically defined ideal

$$
\mathfrak{n}=\left\{v \in A_{t} \text { s.t. } v^{4}=0\right\}=\left(y, x^{2}\right) .
$$

Then

$$
\mathfrak{n} / \mathfrak{m n}=\mathbb{C}\left\langle y, x^{2}\right\rangle \text { and } \mathfrak{n}^{3}=\mathbb{C}\left\langle x^{4} y\right\rangle
$$

Multiplication $\bigodot^{3} \mathfrak{n} / \mathfrak{m n} \rightarrow \mathfrak{n}^{3}$ has the following effect:-

$$
y^{3} \mapsto-\frac{t}{3} x^{4} y \quad y^{2} x^{2} \mapsto \frac{2 t^{2}}{9} x^{4} y \quad y x^{4} \mapsto x^{4} y \quad x^{6} \mapsto-\frac{2 t}{3} x^{4} y .
$$

As polynomial with respect to the dual basis $Y, X$ of $(\mathfrak{n} / \mathfrak{m n n})^{*}$, this is

$$
t Y^{3}-2 t^{2} Y^{2} X-9 Y X^{2}+2 t X^{3}
$$

up to scale. There are no absolute invariants of a binary cubic under $\mathrm{GL}(2, \mathbb{C})$. In fact, all classical invariants are polynomials in a particular invariant $J$ of degree 4 , proportional to the discriminant . However, $\mathfrak{n} / \mathfrak{m} \mathfrak{n}$ has a canonical subspace $\mathfrak{m}^{2} / \mathfrak{m} \mathfrak{n}$ 
spanned by $x^{2}$. Therefore we may look for invariants of this binary cubic under the subgroup of $\mathrm{GL}(2, \mathbb{C})$ consisting of upper triangular matrices

$$
\left(\begin{array}{l}
X \\
Y
\end{array}\right) \mapsto\left(\begin{array}{ll}
a & b \\
0 & d
\end{array}\right)\left(\begin{array}{l}
X \\
Y
\end{array}\right)
$$

The invariant theory for this action is explained in the appendix. In particular, the coefficient of $X^{3}$ defines a linear invariant $K$ and there is a simple quadratic invariant $L$. We may combine these to produce the following absolute invariant:-

$$
\frac{J K^{2}}{L^{3}}=\frac{4 t^{3}}{4 t^{3}+27}
$$

The expression on the right is the $j$-invariant computed by Saito [5]. The replacement $y \mapsto \omega y$ for $\omega^{3}=1$ shows that there are no further invariants.

3.4. Another example. Consider the singularities in $\mathbb{C}^{3}$ given by

$$
V_{t}=\left\{x^{5}+t x^{3} y^{2}+y^{5}+z^{2}=0\right\} \quad \text { for a parameter } t \text { with } 108 t^{5}+3125 \neq 0 .
$$

The moduli algebra may be generated by $x$ and $y$ subject to the relations

$$
x^{4}=-\frac{3 t}{5} x^{2} y^{2} \quad \text { and } \quad y^{4}=-\frac{2 t}{5} x^{3} y .
$$

Iterating these relations quickly leads to

$$
\begin{array}{lrl}
x^{6}=\frac{54 t^{4}}{625} x^{3} y^{3}, & x^{5} y=-\frac{3 t}{5} x^{3} y^{3}, & x^{4} y^{2}=-\frac{18 t^{3}}{125} x^{3} y^{3} \\
x^{2} y^{4}=\frac{30 t^{2}}{125} x^{3} y^{3}, & x y^{5}=\frac{36 t^{4}}{625} x^{3} y^{3}, & y^{6}=-\frac{2 t}{5} x^{3} y^{3}
\end{array}
$$

and so multiplication $\bigodot^{6} \mathfrak{m} / \mathfrak{m}^{2} \rightarrow \mathfrak{m}^{6}$ is represented by the polynomial

$$
\begin{array}{r}
27 t^{4} X^{6}-1125 t X^{5} Y-675 t^{3} X^{4} Y^{2}+6250 X^{3} Y^{3} \\
+1125 t^{2} X^{2} Y^{4}+108 t^{4} X Y^{5}-125 t Y^{6}
\end{array}
$$

up to scale. The invariant theory of homogeneous sextic polynomials in two variables in presented in the appendix. In particular, there is an invariant $J$ of degree 2 and an invariant $K$ of degree 4. A computation (easily carried out with computer algebra, as explained in the appendix) gives

$$
\frac{J^{2}}{J^{2}-2 K}=\frac{78125}{3\left(108 t^{5}+3125\right)}
$$

as an absolute invariant. The replacement $y \mapsto \omega y$ for $\omega^{5}=1$ shows that there are no further invariants.

More generally, the family of singularities given by

$$
f(x, y, z)=x^{5}+s x^{4} y+t x^{3} y^{2}+y^{5}+z^{2}=0
$$

has two absolute invariants derived in a similar way, namely

$$
\frac{\left(3 s t^{2}-125\right)^{2}}{256 s^{5}-1600 s^{3} t-27 s^{2} t^{4}+2250 s t^{2}+108 t^{5}+3125}
$$


and

$$
\frac{\left(\begin{array}{c}
163200 s^{6} t^{2}+14800000 s^{5}-2100000 s^{4} t^{3}+5400 s^{3} t^{6} \\
-92500000 s^{3} t+7425000 s^{2} t^{4}-52650 s t^{7} \\
+116250000 s t^{2}+729 t^{10}-4556250 t^{5}+312500000
\end{array}\right)^{2}}{\left(256 s^{5}-1600 s^{3} t-27 s^{2} t^{4}+2250 s t^{2}+108 t^{5}+3125\right)^{3}} .
$$

Appendix A. We review Weyl's classical theory [7], though most of what we shall need was already well-known in the nineteenth century [2]. Suppose $P\left(X^{1}, \ldots, X^{n}\right)$ is a homogeneous polynomial of degree $N$. Then we may write

$$
p\left(X^{1}, \ldots, X^{n}\right)=\sum_{i, j, \ldots, k=1}^{n} \underbrace{a_{i j \ldots k}}_{N \text { indices }} X^{i} X^{j} \cdots X^{k}=a_{i j \ldots k} X^{i} X^{j} \cdots X^{k}
$$

for a tensor $a_{i j \cdots k}$, symmetric in its indices. In the last expression, the summation is implicit - the Einstein summation convention demands a summation over repeated indices. We shall use this convention form now on. Fix a totally skew tensor $\epsilon^{i \cdots j}$ with $n$ indices. More invariantly, we may view the tensor $a$ as an element of $\bigodot^{N} \mathbb{V}$ for an $n$-dimensional vector space $\mathbb{V}$ and then $\epsilon$ is chosen in $\Lambda^{n} \mathbb{V}^{*}$. Since this space is one-dimensional, $\epsilon$ is unique up to scale. In practise, we can take $\epsilon^{12 \cdots n}=1$ to fix the scale.

As an example, consider the polynomial (3.3). It is homogeneous of degree 3 in 3 variables, classically a 'ternary cubic' - see [2]. Converting to tensor notation in accordance with (A.1), we see that

$$
a_{111}=1, a_{222}=1, a_{333}=1, a_{123}=a_{231}=a_{312}=a_{213}=a_{132}=a_{213}=t / 6
$$

and all other $a_{i j k}$ are zero. Now consider

$$
J=a_{i j k} a_{l m n} a_{p q r} a_{s t u} \epsilon^{i l p} \epsilon^{j m s} \epsilon^{k q t} \epsilon^{n r u} .
$$

Viewing $a$ and $\epsilon$ invariantly, as elements of $\bigodot^{3} \mathbb{V}$ and $\Lambda^{3} \mathbb{V}^{*}$ respectively, it is clear that $J$ is independent of any choice of basis: a summation over repeated indices is simply the invariant contraction $\mathbb{V} \otimes \mathbb{V}^{*} \rightarrow \mathbb{C}$. But $\epsilon^{i j k}$ was chosen arbitrarily and scales by $\operatorname{det}(M)$ under the action of $M \in \mathrm{GL}\left(\mathbb{V}^{*}\right)$. It follows that if we change coördinates $X^{i} \mapsto m^{i}{ }_{j} X^{j}$ in the polynomial $p\left(X^{1}, \ldots, X^{n}\right)$, and recompute $J$, then the answer will be multiplied by $\operatorname{det}\left(m^{i}{ }_{j}\right)^{4}$. Using the original coördinates and choosing $\epsilon^{123}=1$, we find that $J=t\left(t^{3}-216\right) / 54$. Such calculations may be performed on a computer in just a few seconds once a suitable program is written. All the calculations in this article were done this way using the MAPLE computer algebra package. The programs are available electronically ${ }^{1}$.

Expressions such as (A.2), in which various tensors are juxtaposed with all indices paired up, are called 'complete contractions'. Weyl's first fundamental theorem of invariant theory [7] states that all SL $(n, \mathbb{C})$ invariants of any tensor $a_{i j \ldots k}$ arise as linear combinations of complete contractions of $a_{i j \cdots k}$ and $\epsilon^{i \cdots j}$. More complicated complete contractions are best built up from 'partial contractions' (or classical 'covariants' see [2]) such as

$$
b_{i j}{ }^{k l}=a_{p q i} a_{r s j} \epsilon^{p r k} \epsilon^{q s l} \quad c_{i j k}=b_{i j}{ }^{p q} a_{p q k} \quad d^{i j k}=b_{p q}{ }^{i r} a_{r s t} \epsilon^{p t k} \epsilon^{q j s} .
$$

\footnotetext{
${ }^{1} \mathrm{ftp}: / / \mathrm{ftp} . \mathrm{maths}$.adelaide.edu.au/pure/meastwood/maple/README
} 
For example,

$$
J=b_{i j}{ }^{k l} b_{k l}{ }^{i j} \quad \text { and } \quad K=c_{i j k} d^{i j k}
$$

reproduces $J$ as in (A.2) and gives a new invariant $K$ homogeneous of degree 6 . It is a classical theorem [2] that these $J$ and $K$ freely generate the ring of all $\mathrm{SL}(3, \mathbb{C})$ invariants of a ternary cubic.

Theorem A.1. The ratio

$$
j=\frac{J^{3}}{J^{3}-6 K^{2}}
$$

associated to any homogeneous polynomial $p(x, y, z)$ of degree 3 is independent of choice of coördinates. When the denominator is non-zero, this is the $j$-invariant of the elliptic curve $\{p(x, y, z)=0\}$ in $\mathbb{C P}_{2}$.

Proof. Since numerator and denominator are homogeneous of the same degree, namely 12 , a change of coördinates effected by a non-singular matrix $M$ scales both by $\operatorname{det}(M)^{12}$. Though evident by construction, it is also easily checked by direct computation with a computer. In any case, $j$ is coördinate-independent. To show that it is the $j$-invariant, it suffices to verify this for one of the canonical forms of a non-singular cubic. For example, a computer calculation shows

$$
f(x, y, z)=z y^{2}-x(x-z)(x-\lambda z) \Longrightarrow j=\frac{4}{27} \frac{\left(\lambda^{2}-\lambda+1\right)^{3}}{\lambda^{2}(\lambda-1)^{2}} .
$$

This is the well-known formula (normalised as in [5]).

Several canonical forms (including Weierstraß) are included in the computer program 'ternary_cubic' but any polynomial can be treated: for example,

$$
p(x, y, z)=x^{3}+x^{2} y-4 z^{3}+x y z-x z^{2}+x y^{2} \Longrightarrow j=\frac{357911}{120545280} .
$$

This would be difficult to compute if one first had to transform it into a canonical form but using Theorem A.1 and a computer takes just a few seconds. Similarly,

$$
p(x, y, z)=x^{3}+y^{3}+z^{3}+t x y z \Longrightarrow j=-\frac{t^{3}\left(t^{3}-216\right)^{3}}{1728\left(t^{3}+27\right)^{3}} .
$$

This corrects the calculation of $j$ in [5]. On the other hand, it is the reciprocal that computes the $j$-invariant via the moduli algebra and (3.1):-

$$
p(X, Y, Z)=t X^{3}+t Y^{3}+t Z^{3}-18 X Y Z \Longrightarrow \frac{J^{3}-6 K^{2}}{J^{3}}=-\frac{t^{3}\left(t^{3}-216\right)^{3}}{1728\left(t^{3}+27\right)^{3}} .
$$

This completes the invariant theory of the ternary cubic used in $\S 3.1$.

In $\S 3.2$, it was the invariant theory of a homogeneous quartic in two variables that was used. The classical theory of 'binary quartics' is given in Elliott [2]. The ring of invariants is freely generated by

$$
J=b_{i j}{ }^{k l} b_{k l}{ }^{i j} \text { and } K=b_{i j}{ }^{k l} b_{k l}{ }^{m n} b_{m n}{ }^{i j} \text {, where } b_{i j}{ }^{k l}=a_{i j p q} \epsilon^{p k} \epsilon^{q l} .
$$


These are computed in the program 'binary_quartic'. In particular,

$$
p(x, y)=x(x-y)(x-\lambda y) y \Longrightarrow \frac{4 J^{3}}{4 J^{3}-24 K^{2}}=\frac{4}{27} \frac{\left(\lambda^{2}-\lambda+1\right)^{3}}{\lambda^{2}(\lambda-1)^{2}}
$$

so this ratio is the $j$-invariant in general. Then,

$$
p(x, y)=x^{4}+t x^{2} y^{2}+y^{4} \Longrightarrow j=\frac{\left(12+t^{2}\right)^{3}}{108\left(t^{2}-4\right)^{2}},
$$

verifying Saito's result [5]. On the other hand, via the moduli algebra and (3.7):-

$$
p(X, Y)=t X^{4}-12 X^{2} Y^{2}+t Y^{4} \Longrightarrow \frac{J^{3}}{6 K^{2}}=\frac{\left(12+t^{2}\right)^{3}}{108\left(t^{2}-4\right)^{2}},
$$

as claimed in $\S 3.2$.

The invariant theory of the binary sextic was used in $§ 3.4$. As discussed in [2], the ring of invariants has five generators $J, K, L, M$, and $N$ of degrees $2,4,6,10$, and 15 , respectively. To define them, first consider the covariants

$$
\begin{aligned}
& b^{i j k l m n}=a_{p q r s t u} \epsilon^{p i} \epsilon^{q j} \epsilon^{r k} \epsilon^{s l} \epsilon^{t m} \epsilon^{u n} \quad c_{i j}{ }^{k l}=a_{i j p q r s} b^{k l p q r s} \\
& d_{i j}=a_{i j p q r s} c_{t u}^{r s} \epsilon^{p t} \epsilon^{q u} \quad f_{i j}=c_{i j}{ }^{p q} d_{p q} \quad g_{i j}=c_{i j}{ }^{p q} f_{p q} .
\end{aligned}
$$

Then,

$$
\begin{aligned}
& J=c_{i j}{ }^{i j} \quad K=c_{i j}{ }^{k l} c_{k l}{ }^{i j} \quad L=c_{i j}{ }^{k l} c_{k l}{ }^{m n} c_{m n}{ }^{i j} \\
& M=b^{p q r s t u} d_{p q} d_{r s} d_{t u} \quad N=\epsilon^{q r} \epsilon^{s t} \epsilon^{u p} d_{p q} f_{r s} g_{t u} .
\end{aligned}
$$

These are computed in the program 'binary_sextic'. There is just one relation

$$
N^{2}+\frac{1}{1458} J^{15}-\frac{7}{486} J^{13} K+\frac{13}{108} J^{11} K^{2}+\cdots+\frac{1}{8} K L M^{2}+\frac{1}{16} M^{3}=0
$$

given explicitly and verified in binary_sextic. For the purposes of $\S 3.4$, the program checks (3.13) for the polynomial (3.12).

In the case of (3.14), there is an identity $J^{3}+3 J K-10 L=0$ and so we are obliged to use $M$ if we want to construct two absolute invariants in accordance with Theorem 2.1. The two invariants listed for this case are

$$
\frac{3}{5} \frac{J^{2}}{J^{2}-2 K} \text { and } 759375 \frac{M^{2}}{\left(J^{2}-2 K\right)^{5}} .
$$

Finally we discuss the invariant theory behind $\S 3.3$. The basic invariant of a binary cubic $a_{i j k} X^{i} X^{j} X^{k}$ is

$$
J=c_{i}{ }^{j} c_{j}{ }^{i} \quad \text { where } c_{i}{ }^{j}=a_{i p q} \epsilon^{j k} \epsilon^{p l} \epsilon^{q m} a_{k l m} .
$$

It is $-2 / 27$ times the discriminant. The ring of $\mathrm{SL}(2, \mathbb{C})$-invariants consists of polynomials in $J$ so there is no possibility of applying Theorem 2.1 directly. Instead, as suggested in $\S 3.3$, we may look for invariants under the action (3.10) of the upper triangular matrices. Consider the covector $e^{i}$ with $e^{1}=1$ and $e^{2}=0$. It is distinguished by being preserved up to scale by (3.10). Therefore, it is another ingredient that may be used in complete contractions such as

$$
K=a_{i j k} e^{i} e^{j} e^{k} \quad \text { and } \quad L=a_{i j k} a_{l m n} \epsilon^{i l} \epsilon^{j m} e^{k} e^{n}
$$


to produce polynomials that transform by a character under (3.10). Indeed, it is shown in [3] (as a very special case of an invariant theory for tensor representations of parabolic subgroups of the classical groups) that all such polynomials arise as linear combinations of complete contractions like this. In any case, the quotient $J K^{2} / L^{3}$ is not only of polynomials of equal homogeneity but also of the same degree in $\epsilon$, namely 6 . It is, therefore, an absolute invariant of a cubic under (3.10), as required.

\section{REFERENCES}

[1] H. Chen, C. Seeley, And S.S.-T. Yau, Algebraic determination of isomorphism classes of the moduli algebras of $\tilde{E}_{6}$ singularities, Math. Ann., 318 (2000), pp. 637-666.

[2] E.B. Elliott, Algebra of Quantics, Clarendon Press, Oxford, 1895.

[3] C. Fefferman, Parabolic invariant theory in complex analysis, Adv. Math., 31 (1979), pp. $131-262$.

[4] J.N. MATher AND S.S.-T. YAU, Classification of isolated hypersurface singularities by their moduli algebras, Invent. Math., 69 (1982), pp. 243-251.

[5] K. SAito, Einfach-elliptische Singularitäten, Invent. Math., 23 (1974), pp. 281-325.

[6] C. SEeley AND S.S.-T. YAU, Variation of complex structures and variation of Lie algebras, Invent. Math., 99 (1990), pp. 545-565.

[7] H. Weyl, The Classical Groups, Princeton University Press, 1939. 
